\title{
Drag Reduction Characteristics of Bionic Mucous Membrane Acting on the Turbulent Boundary Layer
}

\author{
C. Ma, K. Zhang ${ }^{\dagger}$, B. Zhang, B. Zhao and Q. Wang \\ Department of Mechanical Engineering, College of Engineering, Ocean University of China, Qingdao \\ 266100, PR China
}

†Corresponding Author Email: kszhang@ouc.edu.c

(Received September 15, 2020; accepted February 23, 2021)

\begin{abstract}
The mucous membrane on the fish surface has excellent drag reduction performance. The mucous membrane can be regarded as the viscoelastic fluid, and a bionic friction drag reduction model is proposed with the consideration of a Carreau viscoelastic model-based mucus secretion process. Then, the drag reduction effect of the mucous membrane on the classical wall turbulence boundary layer is investigated by large-eddy simulations. Results show that the bionic mucous membrane is conducive to reducing the turbulence, and can achieve a drag reduction rate of about $14 \%$. This study provides a hydrodynamics understanding of the drag reduction characteristics of the bionic mucous membrane.
\end{abstract}

Keywords: Mucous membrane; Bionic drag reduction; Viscoelastic fluid; Turbulence statistics; Coherent structure

\section{NOMENCLATURE}

$C_{f s} \quad$ drag coefficient of the smooth surface

$C_{f m} \quad$ drag coefficient of the mucous membrane surface

DR drag reduction rate

$H \quad$ characteristic length

I turbulence intensity

$\operatorname{Re} U$ Reynolds number based on $U$

$\operatorname{Re}_{\tau} \quad$ Reynolds number based on $u_{\tau}$

$U$ flow velocity

$U_{i n j} \quad$ mucus injection velocity

$u^{\prime} \quad$ flow direction pulsation velocity

$u_{\tau} \quad$ wall friction velocity

$v^{\prime} \quad$ normal direction pulsation velocity

$X \quad$ flow direction

$\begin{array}{ll}Y & \text { normal direction } \\ Z & \text { span direction } \\ \gamma & \text { shear rate } \\ \lambda & \text { relaxation time } \\ \mu & \text { viscosity of mucus } \\ \mu_{0} & \text { zero shear viscosity } \\ \mu_{\infty} & \text { infinite shear viscosity } \\ \rho & \text { density } \\ \rho_{m} & \text { density of mucus } \\ \tau_{w} & \text { shear stress } \\ v & \text { kinematic viscosity } \\ <> & \text { time average of a variable } \\ ()^{+} & \text {non-dimensionalization of a variable }\end{array}$

In recent years, a large number of bionic friction drag reduction (BFDR) methods have been proposed. The BFDR methods can be divided into active and passive methods. These methods achieve drag-reduction by destroying one or more underlying physical processes that cause surface frictional drag in the turbulent boundary layer (TBL) (Perlin et al. 2016).

Passive BFDR methods can reduce the drag without adding mass, momentum, or energy to the TBL. These methods usually involve changing the shape, chemical or physical properties of the solid boundary 
below the TBL. Passive BFDR is widely used because it is sustainable and rarely wears out. BFDR method for non-smooth surface (Chen et al. 2018; Hou et al. 2020; Liu et al. 2020; Song et al. 2011; Tirandazi and Hidrovo 2020; Heidarian et al. 2018) is one of the earlier BFDR methods. This method is inspired by the shape of fish scales and arranging structures that mimic scales or other patterns on the surface of the object can achieve a drag reduction rate (DR) of up to $10 \%$ on smooth surfaces. The drag reduction mechanism can be explained by the "second vortex theory" or "protruding height theory" (Ke et al. 2009). Besides, researchers have also studied the BFDR method of super-hydrophobic surfaces (SHSs) (Barthlott et al. 2020; Busch et al. 2019; Gu et al. 2017; Liu et al. 2019; Du et al. 2017; Monfared et al. 2019; Saadat-Bakhsh et al. 2017) that imitates hydrophobic surfaces such as lotus leaves (Neinhuis and Barthlott 1997). SHSs are more microscopic than non-smooth surfaces and are usually prepared by laser processing or surface coating methods (Ma et al. 2018; Pan et al. 2016; Wang et al. 2012), which can reach more than $20 \%$ DR at a low flow rate. The "Wall Slip Model" is its current universally recognized drag reduction mechanism (Nosonovsky and Bhushan 2009). Flexible surface (Albers et al. 2020; Huang et al. 2019) is also one of the BFDR methods. A flexible wall was originally produced by imitating the flexible skin of a dolphin. The effect of the flexible wall made the viscous bottom layer thicker, and it reduced the vertical velocity gradient of TBL.

The active BFDR method mainly bases on the use of drag-reduction agent (DRA). It was found that the surface mucus of fish belongs to viscoelastic fluid and has a good drag reduction effect. Since the Toms theory (Toms 1948) was proposed, viscoelastic fluid reduction had aroused the interest of many scholars(Du et al. 2017; Yanuar et al. 2017). Chemical products used as viscoelastic fluids DRA are mainly polymer and surfactant, however, the mechanism of drag reduction is different. In static state, a worm-like micelle structure is formed inside surfactant DRA solution. When there is a shearing force, the worm-like micelle structure tends to align with the flow direction easily, leading to drag reduction effects. Its DR gradually increases as the flow Reynolds number increases, and with reaching a critical point, it decreases with increasing of Reynolds number until to zero (Ge et al. 2007). The long molecular chain structure of the polymer DRA is dissolved in the solvent, and the viscosity of the mixed solution can be significantly improved by increasing the tensile viscosity of the mixed solution. As the viscoelastic relaxation time scale of the fluid is closed to the time scale of the turbulent fluctuations near the wall, the regeneration cycle of the near-wall turbulent flow will be disrupted, resulting in momentum transport perpendicular to the wall and the resulting frictional drag reduction (Zakin et al. 1998). Traditional viscoelastic fluid drag reduction methods are to mix the viscoelastic fluid with the transportation medium to form a mixed solution for drag reduction in pipeline transportation. Tan et al. (2020) used inverse emulsion polymerization to synthesize three DRAs: PHWAM-
1, PHWAM-2, and PHWAM-3. The drag reduction test shows that the three DRAs have different drag reduction properties in freshwater and saltwater due to the different hydrophobic monomers. Gu et al. (2020) discussed the degradation factors of polymer DRA and the anti-degradation performance of surfactants, focusing on the mixed DRA of polymer and surfactant, including mechanism model, drag reduction characteristics, and anti-degradation research. Wang et al. (2020) studied the drag reduction characteristics of the turbulent channel of surfactant DRA with a higher Reynolds number from the perspective of the energy spectrum and multiscale resolution. In the presence or absence of surfactants, two-dimensional vortex proper orthogonal decomposition (POD) is used to perform two-dimensional turbulent flow with Reynolds number, and it is found that pseudo-ordered structures are mainly distributed in the near-wall region, While the number of pseudo-ordered structures in surfactant solution is relatively small. Based on chemical thermodynamics and kinetic analysis, Zhang et al. (2020) phenomenologically explains the generation and loss of drag reduction performance of linear flexible polymers. That is, drag reduction occurs because of an unstable thermodynamic environment, in which free polymers and aggregates lead to a decrease in bulk elongation and drag reduction performance, and broken chains of free polymers and aggregates leads to an unstable dynamic environment. Ling et al. (2020) mixed an anionic polymer (xanthan gum, $\mathrm{XG}$ ) and a cationic surfactant (benzethonium chloride, BC) to form DRA. It is found that the mixed DRA has a stronger drag reduction effect, and the maximum DR of $66 \%$ can be achieved at 300 ppm $\mathrm{BC}$ and $1000 \mathrm{ppm} \mathrm{XG}$ complex.

Viscoelastic fluid additives have been fully affirmed in fluid drag reduction characteristics. However, for the underwater vehicle, the BFDR method of mixing viscoelastic fluid with the main fluid is invalid because it cannot navigate in the mixed solution. Therefore, Zhang et al. (2020) proposed a drag reduction method for mucus secretion on the surface of an underwater vehicle. In this paper, a BFDR model inspired by the mucous membrane on the fish surface is proposed. And the secretion process of mucus represented by the Carreau viscoelastic model(Marrucci 1999) and the drag reduction characteristics of the bionic mucous membrane in the classical wall turbulence boundary layer are investigated by large-eddy simulation, including the turbulent statistics and coherent structure.

\section{MuCous MeMbrane BFDR MODEL}

Figure 1 shows a fluid dynamics simulation model for the study of the mucous membrane BFDR. In this model, the classical wall surface TBL model is the main analysis object of turbulence drag. The upper and lower wall is defined as smooth surface (SS) and mucous membrane surface (MMS) with mucous secretion holes, respectively. The middle point of the lower wall surface at the entrance was taken as the coordinate origin. There are 408 mucus secretion 
holes on the wall. Figure 1(b) shows the distribution of them. Streamwise, normal, and spanwise directions are denoted by $\mathrm{X}, \mathrm{Y}$, and $\mathrm{Z}$. The characteristic length $H=0.005 \mathrm{~m}$. The fluid medium of the flow field is water, the kinematic viscosity is $v=0.000001 \mathrm{~m}^{2} \cdot \mathrm{s}^{-1}$, and the density is $\rho=998.2 \mathrm{~kg} \cdot \mathrm{m}^{3}$.

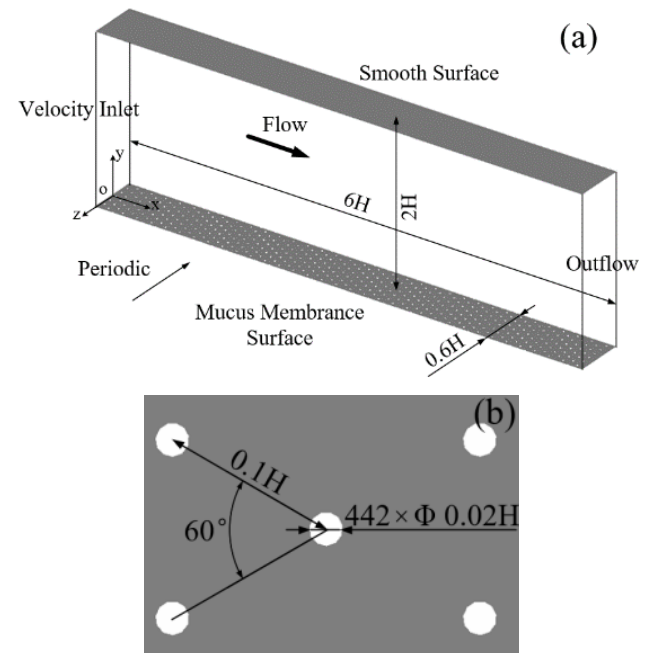

Fig. 1. Mucous membrane BFDR model (a) Flow field and (b) Secretion hole.

Many viscoelastic fluid models can simulate the rheological behavior of viscoelastic fluids, such as Oldroyd-B model (Oldroyd 1950), FENE-P model (Tanner 1975), and Giesekus model (Giesekus 1982), and so on. Most of the viscoelastic fluid drag reduction agent are assumed to be viscoelastic fluid. However, it is more appropriate for the constitutive model of fish mucus to be modeled with the Carreau model. Because the Carreau model fluids behave as Newton fluids at low shear rates, and power-law fluids at high shear rates, which can best fit the rheological parameters of fish mucus. (Gao 2004; Liu et al. 2010; Xu 2018). The Carreau model can be expressed as

$\frac{\mu-\mu_{\infty}}{\mu_{0}-\mu_{\infty}}=\frac{1}{\left[1+(\lambda \gamma)^{2}\right]^{(1-n) / 2}}$

where $\mu_{0}$ represents zero shear viscosity, $\mu_{\infty}$ is infinite shear viscosity, $\lambda$ is relaxation time, $n$ is the power-law coefficient, $\gamma$ is a shear rate, and $\mu$ is the viscosity of mucus. The specific parameters are shown in Table $1\left(\rho_{m}\right.$ represents the density of mucus), which can be input in the software model library.

Table 1 Physical properties of mucus membrane.

\begin{tabular}{|c|c|c|c|c|}
\hline$\mu_{0} / \mathrm{kg} \cdot \mathrm{m}^{-1} \cdot \mathrm{s}^{-1}$ & $\mu_{\infty} / \mathrm{kg} \cdot \mathrm{m}^{-1} \cdot \mathrm{s}^{-1}$ & $n$ & $\lambda / \mathrm{s}$ & $\rho_{m} / \mathrm{kg} \cdot \mathrm{m}^{-3}$ \\
\hline 0.3 & 0.0005 & 0.3 & 50 & 1000 \\
\hline
\end{tabular}

The calculational domain is discretized with polygonal unstructured grids. To accurately describe the turbulent structure near the wall, the normal direction of the fluid domain is refined with unstructured grids, and a boundary layer is set near the wall with the first boundary layer grid height of $y^{+}<1$ (nondimensionalized by $u_{\tau}$.). The velocity inlet boundary is employed for the entrance and the outflow boundary for the exit. The turbulence is periodical in the $Z$-axis direction, where periodic boundary conditions are used. The SS and MMS correspond to the wall without slip.

In this paper, the transient solver of Ansys Fluent2019R3 is adopted to implement the large eddy simulation(LES) of this model. Since the external flow field and mucus can be fused, the Mixture multiphase flow model is employed for the mucous secretion. The pressure-velocity coupled solver and SIMPLEC algorithm are used for calculation, the gradient term is discrete using the Green-Gauss Node Based format, the pressure term is discrete using the PRESTO format and the momentum and volume fraction are both discrete by First Order Upwind format. In order to ensure the accuracy of the calculation, the maximum Courant number of the flow field is set to 2 . According to this, the time step used in this paper is $0.0003 \mathrm{~s}$, which saves calculation time and resources while ensuring that the transient flow field information is not lost. Once the calculation has reached a stable state of turbulence, the statistical quantities are averaged over time.

Figure 2 presents the dependence of the calculated friction coefficient $\left(C_{f}\right)$ on the number of the discretized element of the domain. It is clear that with the increase of the element, the calculated $C_{f}$ gradually stabilizes to the theoretical values obtained from Dean model(Cai 2016). Table 2 lists the relative differences between the numerical and theoretical results. The relative error in $\mathrm{Cf}$ is within $4 \%$ of its value that is predicted by an empirical formula, and the number of grids meets the requirements. It can be concluded that the model with a grid number of $4,236,585$ can ensure calculation accuracy and efficiency, and this model is adopted subsequently.

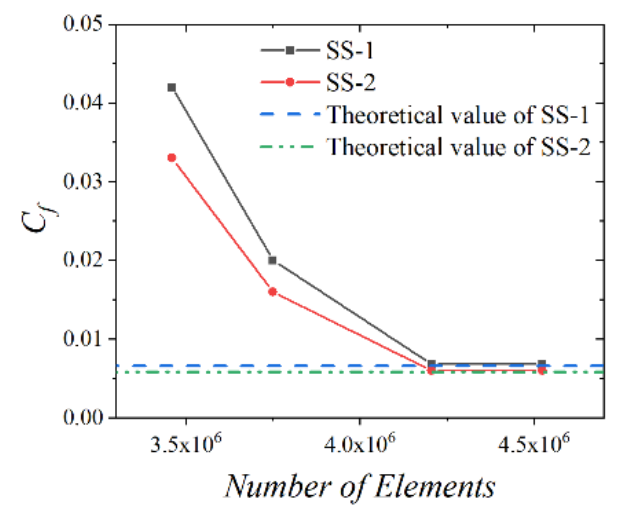

Fig. 2. Relationship between the number of grids and $C_{f}$.

Table 2 Verification of $C_{f}$.

\begin{tabular}{|c|c|c|c|}
\hline \multirow{2}{*}{ Condition } & \multicolumn{2}{|c|}{$C_{f}$} & \multirow{2}{*}{$\begin{array}{c}\text { Relative } \\
\text { Error }\end{array}$} \\
\cline { 2 - 3 } & Theoretical & Simulation & \\
\hline SS-1 & 0.006596 & 0.006830 & $3.55 \%$ \\
\hline SS-2 & 0.005805 & 0.006023 & $3.76 \%$ \\
\hline
\end{tabular}




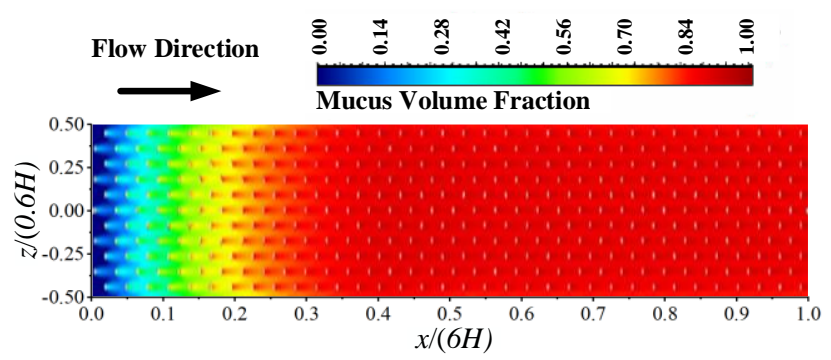

Fig. 3. Mucous membrane distribution.

Table 3 Basic parameters of TBL and $D R$.

\begin{tabular}{|c|c|c|c|c|c|c|c|}
\hline Condition & $U / \mathrm{m} \cdot \mathrm{s}^{-1}$ & $U_{\text {inj }} / \mathrm{m}^{-1} \mathrm{~s}^{-1}$ & $\mathrm{Re}_{\tau}$ & $u_{\tau} / \mathrm{m} \cdot \mathrm{s}^{-1}$ & $\tau_{w} / \mathrm{kg} \cdot\left(\mathrm{m} \cdot \mathrm{s}^{2}\right)^{-1}$ & $C_{f}$ & $D R$ \\
\hline SS-1 & 3 & - & 876.568 & 0.175314 & 30.679525 & 0.006830 & - \\
\hline MMS-1 & 3 & 0.05 & 812.404 & 0.162481 & 26.352473 & 0.005867 & $14.10 \%$ \\
\hline SS-2 & 5 & - & 1371.904 & 0.274381 & 75.149291 & 0.006023 & - \\
\hline MMS-2 & 5 & 0.08 & 1297.837 & 0.259567 & 67.253977 & 0.005390 & $10.51 \%$ \\
\hline
\end{tabular}

\section{DRAg Reduction EFFect OF MuCOUS MEMBRANE}

\subsection{Mucous Membrane Distribution}

The formation of the mucous membrane is the first step for reducing the drag. Figure 3 shows the distribution of the mucous membrane on the wall. At the front end of the flow field, due to the inlet effect of the flow field, the distribution of mucus is very sparse, and the secreted mucus is washed to the back end of the flow field by water. From $x /(6 H)=0$ to $x /(6 H)=0.3$, the mucous membrane gradually formed and the thickness of the mucous membrane gradually increased; After $x /(6 H)=0.3$ the mucous membrane tends to be stable and the thickness is evenly distributed.

\subsection{Average Velocity Profile and DR}

The basic parameters and $D R$ of TBLs on different surfaces have been listed in Table 3. $\operatorname{Re}_{\tau}$ is Reynolds number based on wall friction velocity $u_{\tau}$ defined by Eq. (2). The $C_{f}$ and $D R$ based on shear stress $\tau_{w}$ are given by Eq. (3) (4). (where $C_{f s}$ is the friction coefficient of the SS, $C_{f m}$ is the friction coefficient of the MMS)

$$
\begin{aligned}
& u_{\tau}=\sqrt{\frac{\tau_{w}}{\rho}} \\
& C_{f}=\frac{\tau_{w}}{\frac{1}{2} \rho U^{2}} \\
& D R=\frac{C_{f s}-C_{f m}}{C_{f s}} \times 100 \%
\end{aligned}
$$

It can be seen from Table 3 that the MMS has an excellent $D R$ under two different conditions the case of MMS-1 produces a higher DR (over 14\%). the mucous membrane can reduce the Reynolds number $\operatorname{Re}_{\tau}$ to a certain extent and thus suppress the intensity of turbulence. Figure 4 shows the nondimensional average flow velocity $\left(U^{+}\right)$profile cu rves. Both the classical viscous bottom layer $\left(y^{+}<5\right)$ and the logarithm-law layer curve $\left(30<y^{+}<0.15 \mathrm{Re}_{\tau}\right)$ are also plotted for comparison. It can be seen from Fig. 4 that the velocity profile of the SS-1 and SS-2 are more consistent with the classical distribution of the TBL, which verifies the accuracy of the LES again. Furthermore, the average velocity of the flow field on the MMS is higher than the SS at the viscous bottom layer, the buffer layer, and the logarithm-law layer, and the upward movement of MMS-1 is more significant.
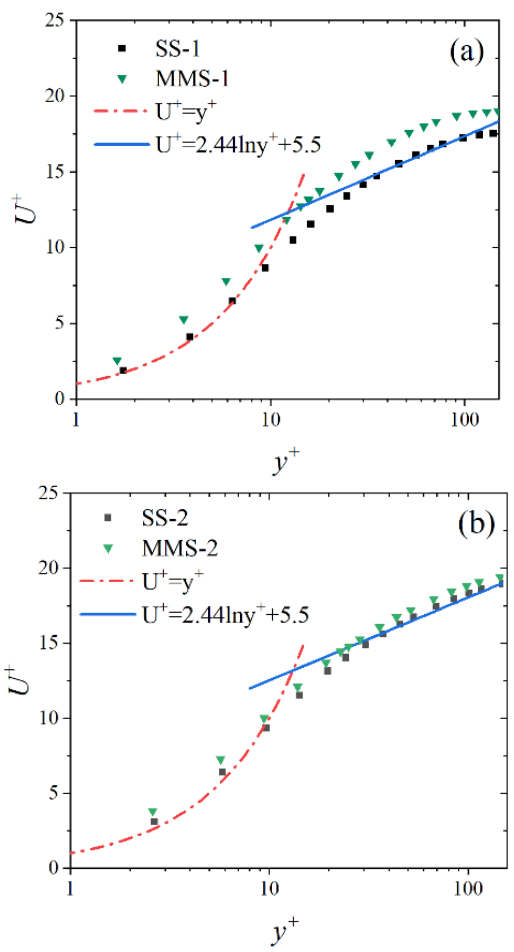

Fig. 4. Average velocity profile (a) SS-1 and MMS-1 conditions; (b) SS-2 and MMS-2 conditions. 


\section{ANAlysis OF DRAg REDUCTION MECHANISM}

In this section, the influence of bionic mucous membrane on turbulence statistics (i.e. the turbulent intensity, and Reynolds stress)are first studied with a series. Then, in the viewpoint of turbulent coherent structure, near-wall streak structure and 3D vortex structure between smooth surface and mucous membrane surface are researched to reveal the drag reduction mechanism of the bionic mucous membrane.

\subsection{Turbulence Statistics}

\section{(a) Turbulence intensity}

Turbulence intensity $(I)$ is used to represent the turbulence strength of the flow field. Figure 5 shows the comprehensive $I^{+}$nondimensionalized by $u_{\tau}^{2}$ comparison between the SS and the MMS under different Re. It can be seen that the $I^{+}$peaks of the two Re all appear near $y^{+}=20$, but MMS has a lower $I^{+}$value. The $I^{+}$on the MMS of the viscous bottom layer $\left(y^{+}<5\right)$ is slightly higher than that of the SS. This is due to the mucus secretion holes have a velocity normal to the wall which causes a certain disturbance to the flow field. This weak disturbance, however, is limited to the viscous bottom layer and cannot offset the powerful drag reduction effect of the mucous membrane.

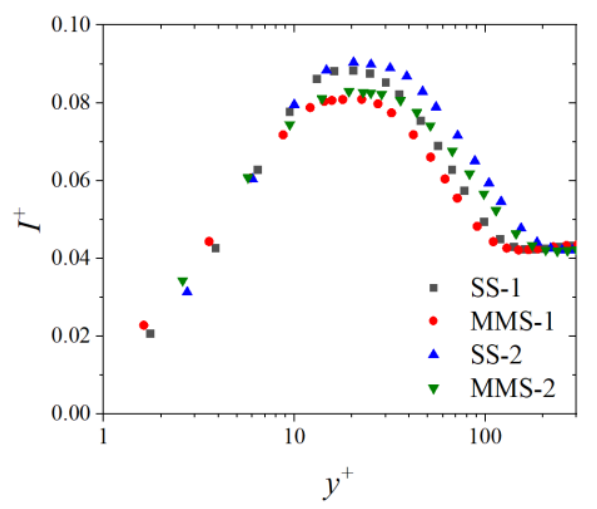

Fig. 5. Turbulence intensity.

\section{(b) Reynolds stress}

Reynolds stress is the additional stress caused by momentum exchange resulting from the turbulent pulsating velocity. In Fig. 6, the distributions of Reynolds normal stress $\left(\left\langle u^{\prime} u^{\prime}\right\rangle^{+}\right)$and Reynolds shear stress $\left(-\left\langle u^{\prime} v^{\prime}\right\rangle^{+}\right)$in normal direction of the SS and the MMS are compared, and both of them are nondimensionalized by $u_{\tau}{ }^{2}$. Under two different Reynolds number conditions, the $\left\langle u^{\prime} u^{\prime}\right\rangle^{+}$of the SS has a peak near the $y^{+}=20$, and the position of $\left\langle u^{\prime} u^{\prime}\right\rangle^{+}$ peak of the MMS is same as that of the SS, but the value is reduced. The SS reaches its peak at the position of $y^{+}=30$, and the peak value of the $-\left\langle u^{\prime} v^{\prime}\right\rangle^{+}$ of the MMS is also reduced, and there is a slight outward shift. The reduction of Reynolds stress is a typical feature of the drag reduction wall. This means that the momentum exchange in the turbulent flow is weakened and the turbulence pulsation is suppressed.
Besides, because of the slight disturbance of the secretion hole entrance velocity, the Reynolds stress of the MMS in the viscous bottom layer is slightly higher than that of the SS.
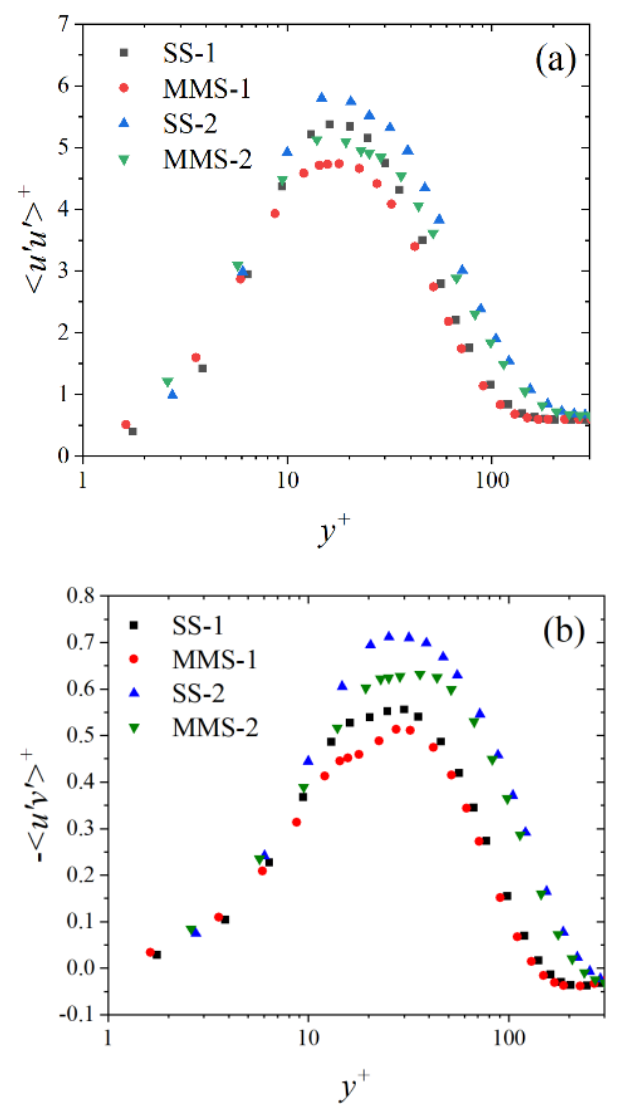

Fig. 6. Reynolds stress (a) Dimensionless Reynolds normal stress and (b) Dimensionless Reynolds shear stress.

\subsection{Coherent Structure}

The classical statistical theory of turbulence considers that the turbulent pulsation is a kind of irregular motion. In the 1950s, Corrdsin (1951) discovered the intermittent phenomenon of velocity pulsation when studying the statistical characteristics of wakes, and speculated that there was a kind of a large-scale structure with special function in turbulent pulsation-coherent structure. This discovery provides a new understanding on the turbulence. Turbulence is not an irregular motion, and among its motions, there are detectable and recognizable coherent motions, which dominate the momentum and energy transport in turbulence.

Coherent structure refers to an ordered movement triggered irregularly in shear turbulence. The turbulent pulsation near the wall is constrained by the wall. It is shown that the small-scale vortex structure near the wall is the most complex and is the primary source of wall drag.(Zhang et al. 2017) The basic elements of a coherent structure are "streak structure" and "vortex structure", which are in a fully developed TBL. A relatively stable cyclic mechanism will be formed. As long as one link in this mechanism is destroyed, the self-sustaining 
process of turbulent coherent structure can be controlled and turbulent bursts can be suppressed (Asrafi et al. 2019).

\section{(a) Streak structure}

Figures 7 and 8 show the resultant velocity distribution at $y^{+}=15$. The velocity streak distribution before and after drag reduction under two different Re was calculated. Because the Reynolds numbers of SS-2 and MMS-2 conditions are much larger than that of SS-1 and MMS-1 conditions, the former is more severe than the latter during the turbulent burst. This severe phenomenon is reflected in the streak structure of SS-2, whose streak structure appeared earlier, and was denser in the entire flow field than SS-1.

In the $X$-axis direction streak structure (Fig. 7(a), $7(\mathrm{~b}), 8(\mathrm{a})$ and $8(\mathrm{~b}))$, there is a high-speed area near the inlet of the flow field. There are almost no streak and turbulence in this area. In the middle of the flow field, the streak structures are gradually formed, with the crossover phenomenon of them becoming increasingly obvious, where is the burst of turbulence, and the turbulence is most intense. At the end of the field, the turbulent tends to be gentle, and the low-speed and high-speed streaks are slender. But there is still an unstable streak crossing. Comparing the $X$-axis direction streak structure before and after drag reduction, under the working conditions of SS- 1 and MMS-1, the turbulence burst on the MMS appeared later than the SS, and the frequency of low-speed streaks was not as dense as the SS. At the end of the flow field, the high-speed streaks on the MMS occupy a dominant position, and the streaks mainly advance along the $X$-axis direction. It is difficult to collide with other streaks to form a $\Lambda$ vortex (See section 4.2 (b)). Under SS-2 and MMS-2 conditions, the streak structure of the MMS still has certain advantages. The turbulent burst on the MMS is delayed, and there are fewer high-speed streaks in the burst. At the end of the flow field, high-speed streaks dominate, and low-speed streaks have fewer disturbance factors.

Figures 7(c), 7(d), 8(c) and 8(d) compare the normal streak structures before and after drag reduction. The normal streak structure reflects the movement of the fluid along the $Y$-axis direction. When the fluid moves along the wall to a certain distance, a normal movement occurs. The streak structure presents a shape elongated in the $Z$-axis direction, and it develops along the $X$-axis direction in a positive and negative pattern. $v>0$ means the fluid is upward sweep; $v<0$ means the fluid is downward sweep, the alternating occurrence of "upward sweep" and "downward sweep" motion promotes the generation of vortex structure and the formation of turbulence. After the turbulence is formed, the flow gradually stabilizes, and the "upward sweep" and "downward sweep" motion no longer appear alternately, but more randomly, and the "upward sweep" phenomenon is dominant. Through comparison of MMS and SS, it is found that the "upward sweep" and "downward sweep" motion of the MMS appears later, which reveals that the mucous membrane delays the burst of turbulence. Moreover, the number
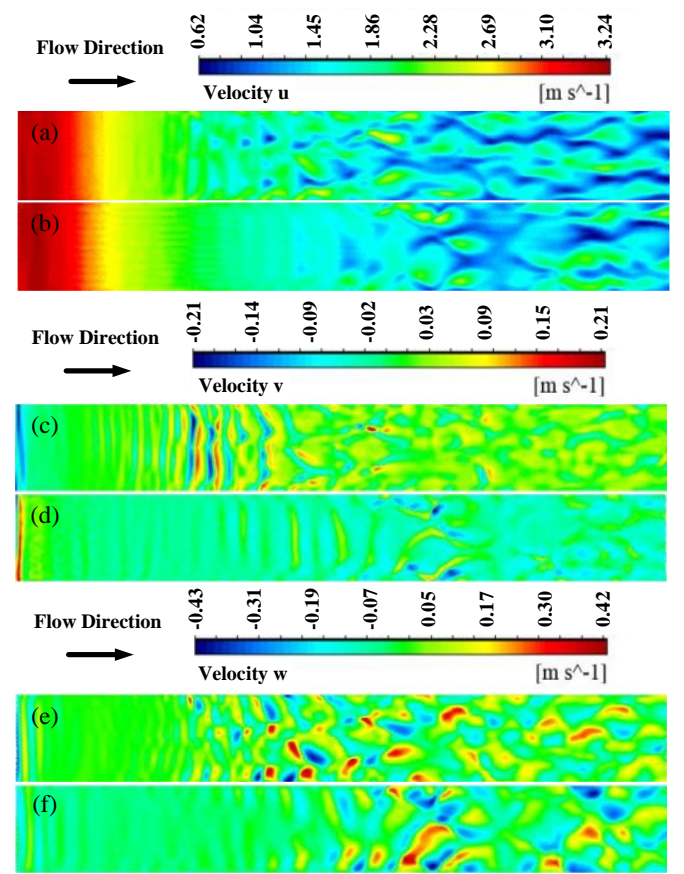

Fig. 7. Streak structure, $\mathrm{Re}=\mathbf{1 5 0 0 0}$, (a) SS-1, $X$ axis direction, (b) MMS-1, $X$-axis direction, (c)

SS-1, $Y$-axis direction, (d) MMS-1, $Y$-axis direction, (e) SS-1, Z-axis direction, (f) MMS-1, $Z$-axis direction.

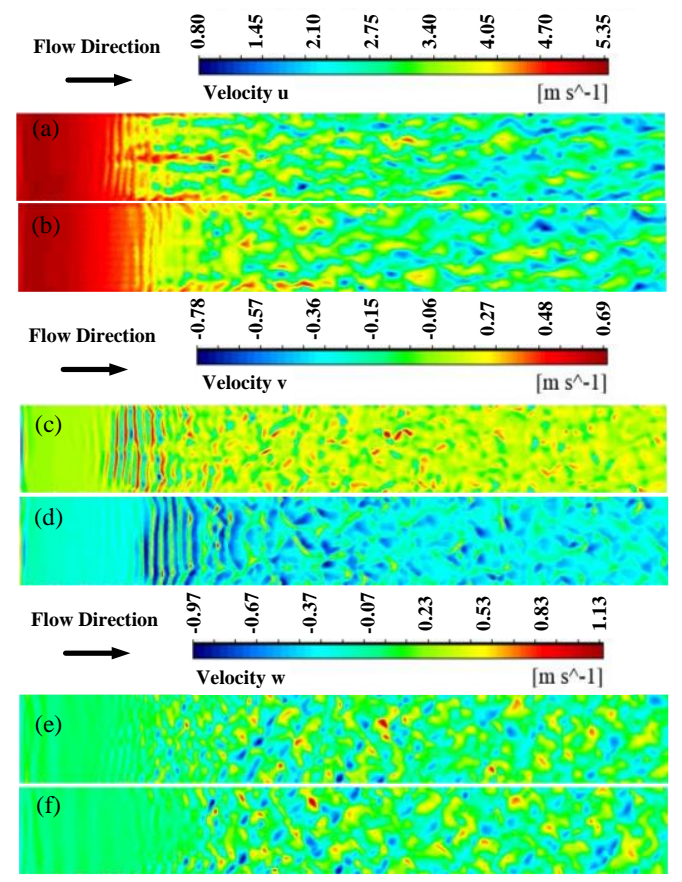

Fig. 8 Streak structure, $\mathrm{Re}=25000$ (a) $\mathrm{SS}-2, X$ axis direction; (b) MMS-2, $X$-axis direction; (c)

SS-2, $Y$-axis direction; (d) MMS-2, $Y$-axis direction; (e) SS-2, Z-axis direction; (f) MMS-2, $Z$-axis direction.

and density of the normal streak structure on the MMS are much smaller than that of the SS, and the normal disturbance is much smaller than the SS. Among them, the contrast effect of SS-1 and MMS1 working conditions is more obvious. 
The spanwise streak structure characterizes the fluid movement along the $Z$-axis direction which is shown in Figs. 7(e), 7(f), 8(e) and 8(f). The span-wise streak structure is unique from the $X$-axis direction and the $Y$-axis direction. In the burst section, a gradually increasing trend from scratch can be found. After the formation of turbulence, the flow shows a positive and negative streak structure. The spanwise pulsation of fluid is also an important factor for fluid drag. After comparison, it reveals that the spanwise streak structure of the MMS appeared later, which is the same size as the streak structure as the SS, but the number was smaller and the density was lower. It indicates that the mucous membrane leads to smaller pulsations in the flow field in the span direction, which can achieve a certain drag reduction effect In a fully developed TBL, the low-speed streaks form a vortex tube under the effect of the flow direction velocity gradient. The vortex tube is induced by the $X$ and $Z$-axis directions to lift and move in the $Z$-axis direction. When two streaks collide, the heads of the streak will join to form a $\Lambda$ vortex.

\section{(b) Three-dimensional vortex structure}

Vortex plays an important role in transporting fluid mass and kinetic energy in turbulent motion. The movement and development of flow direction vortex is an important reason for turbulent burst and drag. At present, the flow vortices that have been found mainly refer to $\Lambda$ vortices. Theodorsen (1952) first proposed the concept of $\Lambda$ vortex, which is shaped like a capital " $\Lambda$ " letter and comprises vortex head with spanwise vorticity, vortex neck, and vortex leg with flow vorticity, as shown in Fig. 9(a). Figure 9(b) is a diagram of the typical $\Lambda$ vortex structure captured by the LES in this study. When the $\Lambda$ vortex moves downstream, the vortex head will develop upwardly at a certain angle along the wall. Because of the adhesion of the vortex legs on the wall, the moving speed of the vortex head is greater than that of the vortex legs, so the $\Lambda$ vortex gradually stretches along the $X$-axis direction. This will increase the vorticity of the $\Lambda$ vortex, causing the fluid inside the vortex head and vortex leg to form a strong upward movement, while the fluid outside the vortex head and vortex leg will sweep down the wall strongly and eventually cause a burst of turbulence.

To model the vortex structure, as well as its evolution and interaction, a $Q$ criterion is adopted to identify the three-dimensional vortex structure in the flow field. The region where of the second invariant of the velocity gradient tensor is positive $(Q>0)$ refers to that there is a swirling motion in the velocity field and a concentrated area in the vorticity field to show the vortex. To better display the vortex structure, the $Q=1.7 \times 10^{6}$ Iso-surface with the velocity contour is selected.

The comparisons of the generation and evolution of the three-dimensional vortex structure under different conditions are shown in Fig. 10. Vortex structure at moments of $0.03 \mathrm{~s}$ and $0.06 \mathrm{~s}$ are compared to study the evolution of the vortex structure. It can be seen that under the conditions of SS-1 and MMS-1, the vortex structure is formed during $0.03 \mathrm{~s}$, and only the front end of the flow field has a vortex structure, and it is in the process of being formed. Because of the mucous membrane, the vortex structure near the entrance is suppressed, and the burst of turbulence is postponed to the middle of the wall. The vortex structure at $0.06 \mathrm{~s}$ is already at a relatively mature stage, and the $\Lambda$ vortex has formed and broken at the tail. The vortex structure of the MMS is much less than that of the SS, which means that the mucous membrane inhibits the generation and development of the vortex structure.
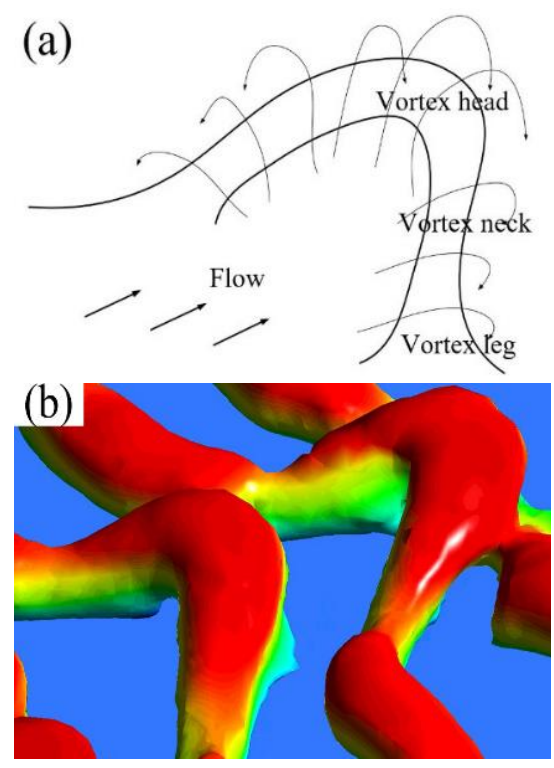

Fig. 9. Vortex structure (a) Schematic diagram of the $\Lambda$ vortex structure (b) The $\Lambda$ vortex structure in this article.

The Reynolds number of SS-2 and MMS-2 conditions is much larger than that of SS-1 and MMS-1 cases, and the vortex structures are also more complicated than those of SS-1 and MMS-1 conditions. At the moment of $0.06 \mathrm{~s}$, a complete $\Lambda$ vortex appears in SS-2 and MMS-2 conditions. The MMS at the end of the wall has an inhibiting effect on the generation of vortex structure. The vortex structures of the two surfaces have been fully developed, but the number of vortex heads on the MMS in the entire watershed( i.e., the number of $\Lambda$ vortices) is less than the that of the SS surface, and there are fewer broken flow vortices, which also affects the vortex structure. Some small vortex packets appear near the secretion holes on the MMS. This is because of the small vortices generated by the flow velocity normal to the wall during mucus secretion, which will cause a slight perturbation of the flow field, the vortex is too small enough to be connected with the flow vortex to form a greater disturbance, and will not prevent the strong suppression of the vortex structure by the mucus membrane.

The mucus membrane not only suppresses the number of vortex structures in the flow field, but also the formation of the vortex structure. Figure 11 shows the typical $\Lambda$ vortex structure where the wall surface is interrupted in SS-2 and MMS-2 conditions. Comparing with the MMS-2 case, overall shape of 


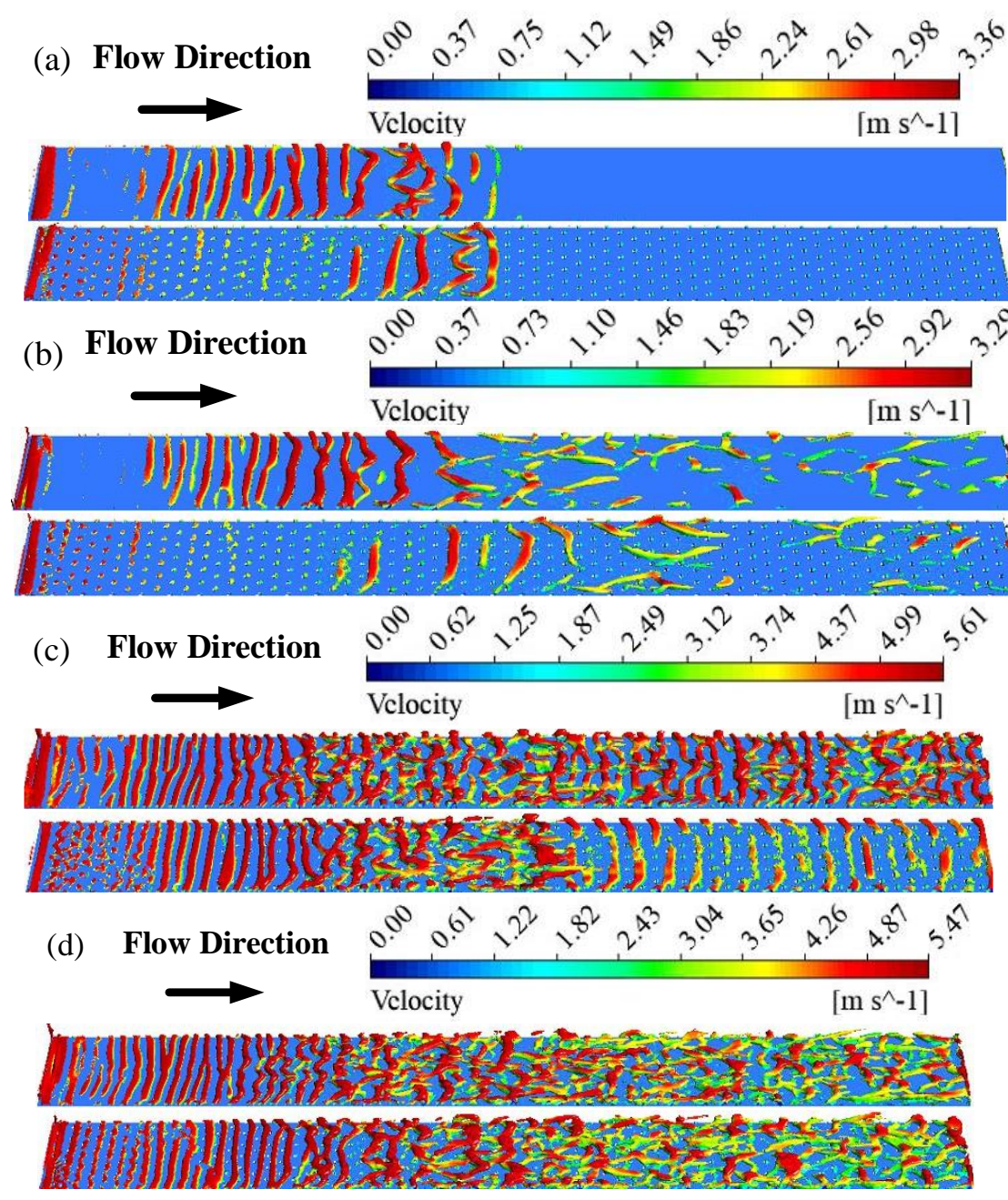

Fig. 10. Three-dimensional vortex structure (a) SS-1 and MMS-1, time: 0.03s ; (b) SS-1 and MMS-1, time: $0.06 \mathrm{~s}$; (c) SS-2 and MMS-2, time: $0.03 \mathrm{~s}$; (d) SS-2 and MMS-2, time: 0.06s ; in all the Figures, the upper side is SS condition and the lower side is MMS condition.
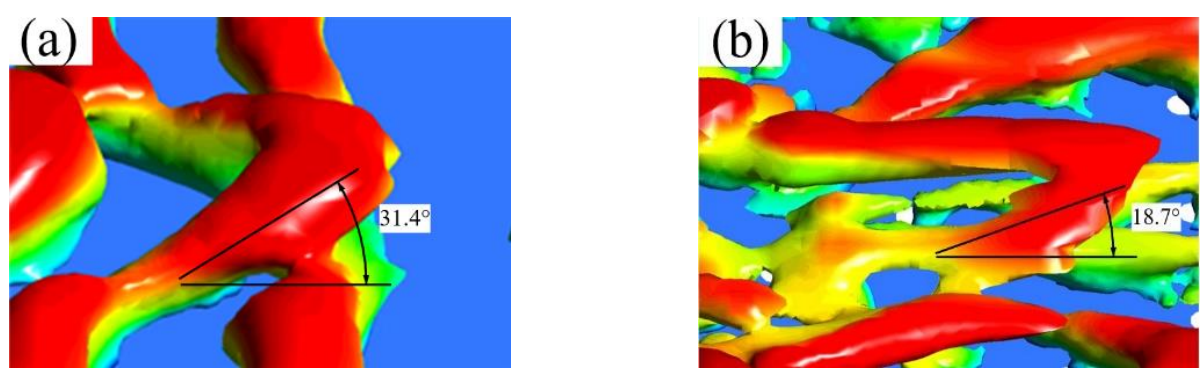

Fig. 11. Comparison of vortex structure between MMS and SS (a) SS-2 condition; (b) MMS-2 condition.

SS-2 is shorter and thicker pruduces, with larger vortex heads and thinner legs. Besides, the SS-2 case also induces a higher vortex head with a lifting angle of about $31.4^{\circ}$. Such a vortex structure makes the SS2 case more likely to be stretched and broken.

\section{CONCLUSION}

The bionic mucous membrane has excellent drag reduction performance, and is very suitable for the underwater vehicle. In this study, a bionic friction drag reduction model is proposed with the consideration of a Carreau viscoelastic model-based mucus secretion process. And the drag reduction characteristics of the bionic mucous membrane are studied by acting it on a turbulent boundary layer.

1. The combination effect of mucus secretion velocity and external flow field velocity can form a mucous membrane on the wall. The mucous membrane is sparsely distributed at 
the inlet of the flow field. After the position of $x /(6 H)=0.3$, the distribution of mucous membrane is stable and uniform.

2. The mucous membrane surface can achieve a DR of over $14 \%$, and the drag reduction effect is better than the current general drag reduction methods mentioned in Introduction, such as groove structure, hydrophobic surfaces or flexible skin.

3. Compared with the Smooth surface, the average velocity profile of the boundary layer of the mucous membrane has moved upward; the turbulence intensity and Reynolds stress are both reduced in the logarithmic layer. The mucus membrane inhibits the formation and development of low-speed streaks and 3D vortex in the flow direction so that the number of low-speed streaks in the fully developed turbulent section is reduced, and the formation of $\Lambda$ vortices became slow, which makes the flow field more regular. Furthermore, the mucus membrane has an inhibitory effect on the vortex structure, which makes it difficult to break and makes it more stable. The wall with vortex structure of this state can achieve drag reduction.

\section{ACKNOWLEDGMENTS}

This work is supported by the Major Basic Research Projects of Natural Science Foundation of Shandong Province, China (No. ZR2020ZD31).

\section{REFERENCES}

Albers, M., P. S. Meysonnat, D. Fernex, R. Semaan, B. R. Noack and W. Schroder (2020). Drag Reduction and Energy Saving by Spanwise Traveling Transversal Surface Waves for Flat Plate Flow. Flow Turbulence and Combustion 105(1), 125-157.

Asrafi, I., M. Yerizam, S. Effendi and A. Mataram (2019). Micro Hydro Electric Power Plant (MHEP) Prototype A Study Of The Effect Of Blade Numbers Toward Turbine Rotational Velocity, Symposium of Emerging Nuclear Technology and Engineering Novelty (Senten 2018) 1198,

Barthlott, W., M. Moosmann, I. Noll, M. Akdere, J. Wagner, N. Roling, L. Koepchen-Thoma, M. A. K. Azad, K. Klopp, T. Gries and M. Mail (2020). Adsorption and superficial transport of oil on biological and bionic superhydrophobic surfaces: a novel technique for oil-water separation. Philosophical Transactions of the Royal Society a-Mathematical Physical and Engineering Sciences 378(2167).

Busch, J., W. Barthlott, M. Brede, W. Terlau and M. Mail (2019). Bionics and green technology in maritime shipping: an assessment of the effect of Salvinia air-layer hull coatings for drag and fuel reduction. Philosophical Transactions of the Royal Society a-Mathematical Physical and Engineering Sciences 377(2138).

Cai, W. H. (2016). Viscoelastic hydrodynamics, Science Press, China.

Chen, W., L. Wang, H. Zhang, G. X. Hou and W. J. Leng (2018). Numerical Simulation of Drag Reduction by Three Dimensional Grooves. Mechanics and Materials Science 37-51.

Corrdsin, S. (1951). The decay of isotropic temperature fluctuations in an isotropic turbulence, Journal of the Aeronautical Sciences 18, 417-423.

Du, P., D. Song, F. Ren, Q. Xue and H. Hu (2017). Flow Characterizations and Drag Reduction on the Hydrophobic Surface with a Full Covering Gas Film. Journal of Applied Fluid Mechanics 10(2), 491-498.

Gao, J. Q. (2004). Surface Characteristics of Cyprinus Carpio Haematoperus Scales and Lubrication Behavior of the Mucus of Misgumus Anguillicaudatus Cantor, Master Thesis, Jilin University, Jilin, China.

Ge, W., Y. Zhang and J. L. Zakin (2007). Surfactant turbulent drag reduction in an enclosed rotating disk apparatus. Experiments in Fluids 42(3), 459-469.

Giesekus, H. (1982). A simple constitutive equation for polymer fluids based on the concept of deformation-dependent tensorial mobility, Journal of Non-Newtonian Fluid Mechanics 11(1-2), 69-109.

Gu, Y. Q., S. W. Yu, J. G. Mou, D. H. Wu and S. H. Zheng (2020). Research Progress on the Collaborative Drag Reduction Effect of Polymers and Surfactants. Materials 13(2).

Gu, Y. Q., W. Q. Zhang, J. G. Mou, S. H. Zheng, L. F. Jiang, Z. Z. Sun and E. Wang (2017). Research progress of biomimetic superhydrophobic surface characteristics, fabrication, and application. Advances in Mechanical Engineering 9(12).

Heidarian, A., H. Ghassemi and P. Liu (2018). Numerical Analysis of the Effects of Riblets on Drag Reduction of a Flat Plate. Journal of Applied Fluid Mechanics 11(3), 679-688.

Hou, Q. M., X. F. Yang, J. Cheng, S. R. Wang, D. R. Duan, J. P. Xiao and W. Y. Li (2020). Optimization of Performance Parameters and Mechanism of Bionic Texture on Friction Surface, Coatings 10(2).

Huang, Q., C. Si, C. Y. Zhao, Y. J. Zhong, K. Deng and Y. J. Hu (2019). The drag reduction performance of low Reynolds number pulsating flow in flexible rectangular channels. Physics of Fluids 31(5).

Ke, G. X., G. Pan, Q. G. Huang, H. B. Hu and Z. Y. Liu (2009). Review of Research on Underwater Drag Reduction Technology. Advances in Mechanics 39(5), 546-554. 
Ling, F. W. M., S. Heidarinik and H. A. Abdulbari (2020). Polymer-surfactant complexes effect on the flow in microchannels: an experimental approach, Chemical Engineering Communications.

Liu, C. B., L. Zhu, J. Li and Y. H. Liang (2019). Fabrication of superhydrophobic bionic surface integrating with VOF simulation studies of liquid drop impacting. Microscopy Research and Technique 82(5), 615-623.

Liu, D. M., X. F. Tao, Y. F. Wang, B. Cai, M. Du and Q. Zheng (2010). Rheological Behavior of Loach Surface Mucus, Acta Polymerica Sinica (04), 468-473.

Liu, W. L., H. J. Ni, P. Wang and Y. Zhou (2020). An investigation on the drag reduction performance of bioinspired pipeline surfaces with transverse microgrooves. Beilstein Journal of Nanotechnology 11, 24-40.

Ma, G. J., X. Liu, W. Zhang and G. Sun (2018). Fabrication and Application of Superhydrophobic Bionic Surface. Rare Metal Materials and Engineering 47(6), 1866-1871.

Marrucci, G. (1999). Rheology of Polymeric Systems, Principles and Applications, American Institute of Chemical Engineers, New York.

Monfared, M., M. A. Alidoostan and B. Saranjam (2019). Experimental Study on the Friction Drag Reduction of Superhydrophobic Surfaces in Closed Channel Flow. Journal of Applied Fluid Mechanics 12(1), 69-76.

Neinhuis, C. and W. Barthlott (1997). Characterization and distribution of waterrepellent, self-cleaning plant surfaces. Annals of Botany 79(6), 667-677.

Nosonovsky, M. and B. Bhushan (2009). Multiscale effects and capillary interactions in functional biomimetic surfaces for energy conversion and green engineering. Philosophical Transactions of the Royal Society a-Mathematical Physical and Engineering Sciences 367(1893), 15111539.

Oldroyd, J. G. (1950). On the Formulation of Rheological Equations of State, Proceedings of the Royal Society of London 200 (1063), 523-541.

Pan, H. H., Z. Wang, W. Z. Fan, C. W. Wang, H. J. Li, F. Bai, J. Qian and Q. Z. Zhao (2016). Superhydrophobic Titanium Surface Micro/Nanostructures Induced by Femtosecond Laster. Chinese Journal of Lasers 43(8), 1-7.

Perlin, M., D. R. Dowling and S. L. Ceccio (2016). Freeman Scholar Review: Passive and Active Skin-Friction Drag Reduction in Turbulent Boundary Layers. Journal of Fluids Engineering-Transactions of the Asme 138(9),

Saadat-Bakhsh, M., N. M. Nouri and H. Norouzi
(2017). On the Mechanism of Drag Reduction in Fully-Developed Turbulent Channel Flow with a Streamwise Micro-featured Superhydrophobic Wall. Journal of Applied Fluid Mechanics 10(5), 1363-1374.

Song, X. W., G. G. Zhang, Y. Wang and S. G. Hu (2011). Use of bionic inspired surfaces for aerodynamic drag reduction on motor vehicle body panels. Journal of Zhejiang UniversityScience A 12(7), 543-551.

Tan, H. Z., J. C. Mao, W. L. Zhang, B. Yang, X. J. Yang, Y. Zhang, C. Lin, J. F. Feng and H. Zhang (2020). Drag Reduction Performance and Mechanism of Hydrophobic Polymers in Fresh Water and Brine. Polymers 12(4).

Tanner, R. I. (1975). Stresses in Dilute Solutions of Bead-Nonlinear-Spring Macromolecules. II. Unsteady Flows and Approximate Constitutive Relations, Journal of Rheology 19(1), 37-65.

Theodorsen, T. (1952). Proceeding of 2nd Midwestern Conference on Fluid Mechanics, Ph.D Thesis, Ohio State University, Ohio, America.

Tirandazi, P. and C. H. Hidrovo (2020). Study of drag reduction using periodic spanwise grooves on incompressible viscous laminar flows. Physical Review Fluids 5(6).

Toms, B. A. (1948). Some observations on the flow of linear Polymer solutions through straight tubes at large Reynolds numbers, Proc Int Congress on Rheology, 135-141.

Wang, H., G. Y. Li, Z. Q. Yang, J. Y. Yao and G. F. Ding (2012). Design and Fabrication of SuperHydrophobic Surface Based on Non-Silicon Micro Machining Technology. Nanotechnology and Precision Engineering 10(3), 273-277.

Wang, L., Z. Y. Zheng, W. H. Cai and F. C. Li (2020). Drag reduction characterizations of turbulent channel flow with surfactant additive by proper orthogonal decomposition and wavelet transform. Korea-Australia Rheology Journal 32(1), 1-14.

Wisniewski, P., F. Balduzzi, Z. Bulinski and A. Bianchini (2020). Numerical Analysis on the Effectiveness of Gurney Flaps as Power Augmentation Devices for Airfoils Subject to a Continuous Variation of the Angle of Attack by Use of Full and Surrogate Models. Energies 13(8), 1877.

$\mathrm{Xu}$, C. (2018). Measurement of rheological properties of puffer surface mucus and research on the application of bionic drag reduction, Master Thesis, Jiangsu University Of Science And Technology, Jiangsu, China.

Yanuar, Gunawan, K. T. Waskito and S. Ogata (2017). Effect of Agar Jelly Coating in Rectangular Pipe to Flow Drag Reduction. Journal of Applied Fluid Mechanics 10(4), 1161-1166. 
C. Ma et al. / JAFM, Vol. 14, No. 5, pp. 1317-1327, 2021.

Zakin, J. L., B. Lu and H. W. Bewersdorff (1998). Surfactant Drag Reduction, Rev. Chem. Eng 14(5), 253-320.

Zhang, W. H., H. N. Zhang, J. F. Li, B. Yu and F. C. $\mathrm{Li}$ (2020). Comparison of turbulent drag reduction mechanisms of viscoelastic fluids based on the Fukagata-Iwamoto-Kasagi identity and the Renard-Deck identity. Physics of Fluids 32(1).

Zhang, Z. S., G. X. Cui, C. X. Xu and W. X. Huang (2017). Theory and Modeling of Turbulence, Tsinghua University Press, Beijing, China. 